\title{
Personalisierte Therapie durch Nachweis genetischer Mutationen
}

\author{
Auch in der Parkinson-Therapie könnte in Zukunft die \\ „personalisierte Medizin“ an Bedeutung gewinnen. \\ Neue therapeutische Konzepte setzten bisher vor allem \\ bei bestimmten genetischen Mutationen an.
}

Parkinson-Patienten mit Nachweis einzelner Mutationen bieten erste Ansatzpunkte für ein individuelles Management der Erkrankung, sagte Dr. Susan Fox aus Toronto, Kanada. Patienten mit einer PARKIN-Mutation sprechen in der Regel gut auf L-Dopa an - entwickeln aber früh Dyskinesien. Eine Demenzentwicklung wird bei diesen Patienten nicht beobachtet. Dahinter steckt eine mitochondriale Dysfunktion, was zukünftig Ansatzpunkte für neue Therapien liefern könnte.

Die LRRK2-Mutationen zeigen ein heterogenes Bild. Die Mutation GS2019S ist durch bessere motorische Scores und geringere Depressivität im Vergleich zur sporadischen Form gekennzeichnet - GS2385R durch vermehrte motorische Fluktuationen. Bei beiden Mutationen findet man gehäuft einen durch Akinese und Rigidität gekennzeichneten Subtyp. Zurzeit seien mehrere LRRK2-Inhibitoren in präklinischer Entwicklung. Viele neue Therapien setzten an der vermehrten Expression und Akkumu- lation von $\alpha$-Synuclein an. Hier sei noch unklar, ob alle Parkinson-Patienten von solchen Therapien profitieren oder nur Träger entsprechender Mutationen, sagte Fox. Zurzeit werden eine aktive und passive Immunisierung sowie $\alpha$-Synuclein-Inhibitoren in klinischen Phase-I- und II-Studien getestet. Ein weiterer Ansatz sind Mutationen im Glucocerebrosidase(GBA)-Gen, die bei etwa $10 \%$ der Parkinson-Patienten nachweisbar sind. Diese Patienten unterscheiden sich in Bezug auf die motorischen Symptome nicht vom sporadischen Morbus Parkinson, entwickeln aber mehr kognitive Defizite. Durch die verminderte Aktivität von GBA kommt es hier zu einem Anstieg der $\alpha$-SynucleinSpiegel. Eine Steigerung der GBA-Aktivität - möglich etwa durch Ambroxol - könnte somit eine Therapiestrategie sein.

Ganz am Anfang stehe man noch bei pharmokogenetischen Untersuchungen. Für Rasagilin wurde bei früher Parkinson-Erkrankung gezeigt, dass Patienten mit bestimmten Mutationen im Dopamin-D2-Rezeptor-Gen (rs2283265 und rs1076560) besonders gut auf die Therapie ansprechen.

Maria Weiß

Symposium „Stratifying Parkinson's Disease(s): Implications for Diagnosis and Treatment", 20. International Congress of Parkinson's Disease and

Movement Disorders, 20.6.2016, Berlin

\section{REM-Schlaf-Verhaltensstörung liefert wichtige Hinweise für frühe Diagnosestellung}

\begin{abstract}
Viele Strategien zur Neuroprotektion bei idiopathischem Parkinson-Syndrom sind gescheitert - wahrscheinlich, weil sie viel zu spät kommen. Um die Nervenzellen effektiv vor der Degeneration zu schützen, muss man möglicherweise schon vor dem Auftreten der typischen motorischen Symptome ansetzen.
\end{abstract}

Einige nicht motorische Symptome des Morbus Parkinson treten bereits lange Zeit vor Manifestation der motorischen Symptome auf und könnten somit einen Ansatzpunkt für eine frühzeitige Diagnose und Therapie liefern, sagte Professor Wolfgang Oertel, Klinik für Neurologie an der Universität Marburg. Dazu

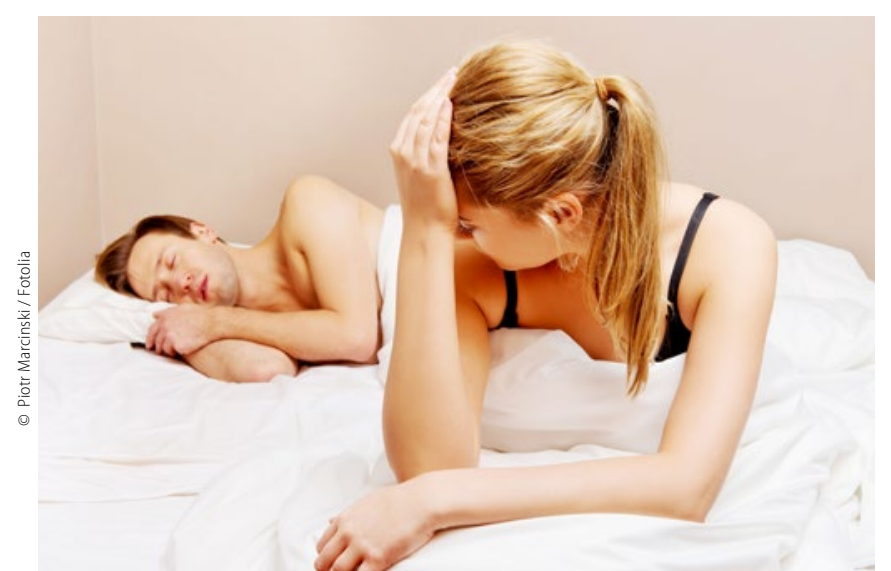

gehören Obstipation, Hyposmie oder Ansomie, REM-SchlafVerhaltensstörung (RBD) oder auch eine Akinesie. Das Problem ist, dass die meisten dieser Symptome völlig unspezifisch sind und sich daher wenig für ein Screening auf eine frühe Parkinson-Erkrankung eignen. Anders ist die Situation bei der RBD: Das motorische Ausagieren von Träumen ist ein relativ seltenes Symptom und $85 \%$ der Betroffenen entwickeln innerhalb von 15 Jahren eine Parkinson-Erkrankung. Durch wenige einfache Fragen an den Partner des Patienten (Schnarcht ihr Partner? Spricht er im Schlaf? Hat er Sie schon mal im Schlaf geschlagen?) lässt sich schon eine Verdachtsdiagnose stellen, mit der man 30-50\% der Patienten mit späterer Entwicklung einer Parkinson-Erkrankung erkennt, sagte Oertel. In der Polysomnografie kann der Befund dann bestätigt werden.

In dieser RBD-Kohorte könnten dann noch weitere zusätzliche Parameter für eine frühe Diagnose herangezogen werden. Dazu gehören zum Beispiel Hyposmie, Obstipation, gestörte Magenmotilität, spezifische Muster im ${ }^{18} \mathrm{~F}$-FluordesoxyglucosePET, veränderte Pupillenreaktionen und eine genetische Charakterisierung. Mit solchen Kombinationen könnte in Zukunft eine frühzeitige Diagnose möglich sein, die eine frühe neuroprotektive Therapie rechtfertigt.

Maria Weiß

Symposium "Late-Breaking Advances in Clinical, Basic and Translational Science", 20. International Congress of Parkinson's Disease and Movement Disorders, 21.6.2016, Berlin 